\title{
HPLC Analysis and Antioxidant Evaluation of Acteoside-Rich Osmanthus fragrans Extracts
}

\author{
Sung-Ho Lee, ${ }^{1,2}$ Seong-Wook Seo, ${ }^{3}$ Seung-Yub Song, ${ }^{1}$ Deuk-Sil Oh, ${ }^{4}$ Jung-Hyun Shim, ${ }^{1,2}$ \\ Goo Yoon, ${ }^{1}$ Ye-Yong Choi, ${ }^{5}$ Do-Kyung Kim, ${ }^{6}$ Jae-Sung Kim, ${ }^{6}$ In-Soo Yoon $\mathbb{D},{ }^{3}$ \\ and Seung-Sik Cho ${ }^{1,2}$ \\ ${ }^{1}$ College of Pharmacy, Mokpo National University, 1666 Yeongsan-ro, Muan-gun, Jeollanam-do 58554, Republic of Korea \\ ${ }^{2}$ Department of Biomedicine, Health \& Life Convergence Sciences, BK21 Four, College of Pharmacy, Mokpo National University, \\ Jeonnam 58554, Republic of Korea \\ ${ }^{3}$ College of Pharmacy, Pusan National University, 2 Busandaehak-ro 63beon-gil, Geumjeong-gu, Busan 46241, Republic of Korea \\ ${ }^{4}$ Jeollanam-do Forest Resource Research Institute, 7 Dado-ro, Sanpo-myeon, Naju-si, Jeollanam-do 58213, Republic of Korea \\ ${ }^{5}$ Research and Development Institute, CY Pharmaceutical Co., 340 Nonhyeon-ro, Gangnam-gu, Seoul 06227, Republic of Korea \\ ${ }^{6}$ School of Dentistry, Chosun University, Gwangju, 460 Iksan-daero, Iksan-si, Jeollabuk-do 54538, Republic of Korea
}

Correspondence should be addressed to In-Soo Yoon; insoo.yoon@pusan.ac.kr and Seung-Sik Cho; sjason1@naver.com

Received 16 April 2020; Revised 7 August 2020; Accepted 13 August 2020; Published 2 September 2020

Academic Editor: Seyed Mohammad Taghi Gharibzahedi

Copyright (C) 2020 Sung-Ho Lee et al. This is an open access article distributed under the Creative Commons Attribution License, which permits unrestricted use, distribution, and reproduction in any medium, provided the original work is properly cited.

A method for the separation and quantification of acteoside by reverse-phase high-performance liquid chromatography (HPLC) was developed and validated. Hot water and ethanolic extracts of Osmanthus fragrans leaves and flowers were analyzed for acteoside content. Excellent linearity was obtained, with an $r^{2}$ higher than 0.999 . The precision, specificity, and accuracy of our method were excellent, suggesting that it can be conveniently used for the quantification of acteoside in the crude extract of $O$. fragrans. The hot water and ethanol extracts were analyzed, and their biological activities were tested. The extraction yields, marker (acteoside) contents, and antioxidant activities of the leaf and flower extracts were analyzed. The antioxidant activity was confirmed by measuring the 2,2-diphenyl-2-picrylhydrazyl radical scavenging activity, reducing power, and total phenolic content. The acteoside content tended to be higher in the $100 \%$ ethanol extract of $O$. fragrans compared to those with the other extraction conditions tested. Overall, almost all extracts prepared with ethanolic solvents tended to produce better antioxidant activity than those prepared with hot water. These results suggest that the ethanolic extract of $O$. fragrans could serve as a potential antioxidant and anti-inflammatory pharmaceutical source, and our validated method would be useful for the quality control of O. fragrans extracts.

\section{Introduction}

Osmanthus fragrans (Oleaceae) is a well-known ornamental plant in Asia [1]. It is widely cultivated as an ornamental plant for its fragrant flowers in Korea, Taiwan, Japan, China, and elsewhere. O. fragrans has been used for treating weakened vision, asthma, coughs, toothaches, stomachaches, diarrhea, and hepatitis. However, modern evidence for the biomedical use of O. fragrans is limited [2]. Recently, various biological activities of $O$. fragrans, such as antilipotoxic [3], antihypoxic [4], anti-inflammatory [5], antiallergic [2], and neuroprotective [6] activities, were reported. Zhou et al. reported that the extract of $O$. fragrans flowers showed antihypoxic activity, and $80 \%$ acetone and 95\% ethanol (EtOH) extracts contained about 3-7\% acteoside (Figure 1) [4]. Hung et al. reported that the $75 \%$ EtOH extract of $O$. fragrans flowers had an antiallergic effect in a mouse model at an oral dose of $1 \mathrm{~g} / \mathrm{kg} / 14$ day [2]. According to their results, the extract of $O$. fragrans flowers could be administered at a dose of about $4.8 \mathrm{~g} /$ day to a $60 \mathrm{~kg}$ human. However, pharmacologically active substances supporting their results have not been reported; only total 
<smiles>C[C@@H]1O[C@@H](O[C@@H]2[C@@H](O)[C@H](O)[C@@H](O)[C@H](O)[C@H]2OC(=O)/C=C/c2ccc(O)c(O)c2)[C@H](CO)O[C@H]1OCCc1ccc(O)c(O)c1</smiles>

Figure 1: Chemical structure of acteoside (verbascoside).

phenolic compounds and total flavonoid concentrations have been reported $(367 \mathrm{mg} / \mathrm{g}$ phenolics and $45 \mathrm{mg} / \mathrm{g}$ flavonoids) [7].

Few studies have reported the possibility of medicinal applications of $O$. fragrans. Recently, Lu et al. reported a study showing that extracts of $O$. fragrans flowers had very low toxicity, suggesting that $O$. fragrans flowers could be developed as a natural drug [1]. They used $80 \% \mathrm{EtOH}$ for the extraction and partially purified the extract with adsorptive macroporous resin to prepare an extract containing $52 \%$ acteoside. Extracts of $O$. fragrans flowers showed no toxicity for 90 days at a dose of $2 \mathrm{~g} / \mathrm{kg} /$ day. This is equivalent to 90 days of administration of $1 \mathrm{~g} / \mathrm{kg}$ as acteoside. Based on these results, the acteoside dose calculated for humans is $4.86 \mathrm{~g} / 60 \mathrm{~kg}$ [1]. The pharmacological activity of $O$. fragrans has been reported in several studies. However, there is a lack of research on the origin of the source materials and their association with biologically active compounds. Recently, researchers have reported the biological activities and identified several components of the flower extracts of $O$. fragrans. Hung et al. identified five compounds, including tyrosyl acetate, (+)-phillygenin, (8E)-ligustroside, rutin, and acteoside from the dichloromethane extract of $O$. fragrans flowers [7]. Among them, phillygenin, rutin, and acteoside showed strong antioxidant activity. Liu et al. identified 16 compounds from $O$. fragrans var aurantiacus flowers; among them, ligustroside and (+)-pinoresinol were identified as showing the best inhibition of nitric oxide production in lipopolysaccharideinduced RAW 264.7 cells [8].

We reviewed the results of previous studies extensively and confirmed that acteoside is a major component of $O$. fragrans and that $O$. fragrans could be developed as a safe medicinal source. However, the flower parts of $O$. fragrans are hard to industrialize owing to low yields. In a preliminary study, we found that the main component of O. fragrans was acteoside and that it was present in large amounts in the flowers and leaves. In the present study, we established a quality control method by high-performance liquid chromatography (HPLC), which could separate and quantify acteoside in the extracts of $O$. fragrans leaves and flowers. To obtain an acteoside-rich extract, leaf and flower extracts were prepared from O. fragrans and O. fragrans var. aurantiacus, which are distinctive plants grown in Korea. Subsequently, the extraction yields, acteoside contents, and antioxidant activities of the leaf and flower extracts were examined. Antioxidant activity was confirmed by measuring the 2,2-diphenyl-2-picrylhydrazyl (DPPH) radical scavenging activity, reducing power, and total phenolic content.

\section{Materials and Methods}

2.1. Plant Materials. Leaves and flowers of O. fragrans and O. fragrans var. aurantiacus were supplied by the Jeollanamdo Forest Resource Research Institute (Naju, Korea). A voucher specimen (MNUCSS-OF-01 04) was deposited at the Mokpo National University (Muan, Korea). Air-dried and powdered $O$. fragrans leaves and flowers $(20 \mathrm{~g})$ were subjected to extraction twice with $20,40,60,80$, and $100 \%$ $\mathrm{EtOH}(100 \mathrm{~mL})$ at room temperature for $48 \mathrm{~h}$ or subjected to extraction with hot water $\left(100^{\circ} \mathrm{C}\right)$ for $4 \mathrm{~h}$. The extraction was repeated twice. The resultant solution was evaporated, dried, and stored at $-20^{\circ} \mathrm{C}$ for further experiments.

2.2. Identification of Acteoside in O. fragrans Extracts. All analyses were performed on a Waters Alliance 2695 HPLC system with a photodiode array detector. The analytical column used was an Agilent Zorbax extended C18 $(5 \mu \mathrm{m}$, $150 \mathrm{~mm} \times 5 \mathrm{~mm}$ ), with the mobile phase consisting of a mixture of solvent A (acetonitrile) and B (water containing $0.2 \%$ phosphoric acid) and employing a gradient elution (from $10 / 90$ to $100 / 0, \mathrm{v} / \mathrm{v}$ ) at a flow rate of $1 \mathrm{~mL} / \mathrm{min}$ (Table 1 ). The column temperature was maintained at $25^{\circ} \mathrm{C}$, and the detection wavelength was set at $270 \mathrm{~nm}$ for acteoside [ $(2 R, 3 R, 4 R$, $5 R, 6 R)$-6-[2-(3,4-dihydroxyphenyl)ethoxy]-5-hydroxy-2-(hydroxy-methyl)-4-[(2S, 3R, 4R, 5R, 6S)-3,4,5-trihydroxy-6methyloxan-2-yl]oxyoxan-3-yl] (E)-3-(3,4-dihydroxy -phenyl) prop-2-enoate.

The acteoside standard was purchased from ChemFaces Biochemical Co., Ltd. (Wuhan, China) and had a purity of $>98 \%$ (Figure 1).

2.3. Method Validation. For the validation of the analytical method used for the quantification of acteoside in the extract of O. fragrans, specificity, linearity, sensitivity, accuracy, precision, and recovery were determined as previously described [9].

2.4. DPPH Free Radical Assay. Antioxidant activity was determined using a DPPH radical-scavenging assay. In brief, $1 \mathrm{~mL}$ of sample solution was mixed with DPPH sample solution $(0.4 \mathrm{mM})$. The mixture contents were allowed to react at room temperature for $10 \mathrm{~min}$. The absorbance of the mixture at $517 \mathrm{~nm}$ was then measured using a microplate reader (Perkin Elmer, Waltham, MA, USA) [10].

2.5. Ferric-Reducing Antioxidant Power (FRAP) Test. The reducing power of the sample was determined using a modified FRAP test [10]. In brief, the sample was mixed with sodium phosphate buffer $(0.2 \mathrm{M})$ and potassium ferricyanide $(1 \%, \mathrm{w} / \mathrm{v})$ followed by incubation at $50^{\circ} \mathrm{C}$ for $20 \mathrm{~min}$. Subsequently, trichloroacetic acid solution $(10 \%, \mathrm{w} / \mathrm{v})$ was added 
TABLE 1: Analytical conditions of high-performance liquid chromatography for the analysis of acteoside.

\begin{tabular}{lcc}
\hline Time $(\min )$ & A & B \\
\hline 0 & 10 & 90 \\
7 & 10 & 90 \\
18 & 100 & 0 \\
19 & 10 & 90 \\
30 & 10 & 90 \\
\hline
\end{tabular}

A: acetonitrile; B: $0.2 \%$ phosphoric acid.

to the reaction mixture followed by centrifugation at $12000 \times \mathrm{g}$ for $10 \mathrm{~min}$. The supernatant was mixed with distilled water and iron (III) chloride solution (0.1\%). The absorbance of the resulting solution was measured at $700 \mathrm{~nm}$. The reducing power of the samples is expressed as ascorbic acid equivalents.

2.6. Determination of Total Phenolic Content. Total phenolic content was determined using the Folin-Ciocalteu assay. The sample was mixed with sodium carbonate solution $(2 \%$, w/v) and Folin-Ciocalteu's phenol reagent (10\%, v/v) for $10 \mathrm{~min}$, and its absorbance was measured at $750 \mathrm{~nm}$. The data are expressed as milligrams of gallic acid equivalents per gram of sample [10].

2.7. Statistical Analysis. The $t$-test was used for comparing two unpaired means, and a $p$ value $<0.05$ was considered statistically significant. Data are expressed as mean \pm standard deviation.

\section{Results and Discussion}

3.1. Extraction Yields of $O$. fragrans Extracts. To date, there have been no reports on yields of extracts prepared from O. fragrans leaves (OFL), O. fragrans flowers (OFF), $O$. fragrans var aurantiacus leaves (OFVL), and O. fragrans var aurantiacus flowers (OFVF). As shown in Table 2, the hot water and $20-100 \%$ EtOH extraction yields ranged from $6.12 \%$ to $42.22 \%$. The best overall yield was obtained by the hot water extraction of $O$. fragrans flowers. This study is the first report of the extraction yields determined according to the extraction conditions for two species of $O$. fragrans. Our data could provide optimal extraction information when O. fragrans is developed as a pharmaceutical material in the future.

\subsection{Method Validation}

3.2.1. Limit of Detection (LOD), Limit of Quantification (LOQ), and Linearity. The LOQ of an individual analytical procedure is the lowest amount of analyte in a sample that can be determined with suitable precision and accuracy. The LOD and LOQ for acteoside were found to be 0.26 and $0.78 \mu \mathrm{g} / \mathrm{mL}$, respectively. The LOD and LOQ values derived from this experiment were less than $1 \mu \mathrm{g} / \mathrm{mL}$ and were sufficient for the analysis of $O$. fragrans. The calibration curve was linear over an acteoside concentration range of
TABLE 2: Extraction yields of the Osmanthus fragrans leaves (OFL), $O$. fragrans flowers (OFF), O. fragrans var. aurantiacus leaves (OFVL), and O. fragrans var. aurantiacus flowers (OFVF) $(n=1)$.

\begin{tabular}{lcccc}
\hline & OFL & OFF & OFVL & OFVF \\
\hline Hot water & 11.93 & 42.22 & 17.67 & 25.71 \\
$20 \% \mathrm{EtOH}$ & 8.41 & 11.00 & 14.89 & 15.53 \\
$40 \% \mathrm{EtOH}$ & 16.53 & 16.66 & 14.67 & 18.59 \\
$60 \% \mathrm{EtOH}$ & 15.68 & 17.44 & 14.44 & 17.76 \\
$80 \% \mathrm{EtOH}$ & 15.67 & 17.13 & 12.41 & 15.88 \\
$100 \% \mathrm{EtOH}$ & 9.53 & 6.12 & 6.99 & 11.23 \\
\hline
\end{tabular}

$6.25-100 \mu \mathrm{g} / \mathrm{mL}$. The calibration curves exhibited good linear regression $\left(r^{2}=0.999\right)$ (Table 3 ).

3.2.2. Precision, Accuracy, and Repeatability. The intraday and interday precision and accuracy data are shown in Table 4 . The developed method was found to be precise, with the obtained RSD values being below $2.0 \%$, and the accuracy was in the range of $98.33-103.42 \%$. The recovery data are shown in Table 5 . These results indicate that the developed method was reproducible with good precision and accuracy.

3.3. Acteoside Contents in O. fragrans Extracts. The acteoside contents in the $O$. fragrans extracts were determined by HPLC analysis. As shown in Figure 2, acteoside was identified as the main compound in the extract of $O$. fragrans. We will identify other bioactive substances in this plant in the future. In the present study, we used acteoside as the main target, and tried to develop an acteoside-rich extract as a drug.

The acteoside contents resulting from various extraction conditions are shown in Figure 3. The acteoside content of the $O$. fragrans extracts tended to be significantly higher in ethanolic extracts than in hot water extracts. All three types of extracts, except OFL, showed more than 10\% acteoside content in the $100 \% \mathrm{EtOH}$ extract. In particular, the content of the $100 \%$ ethanol extract of OFVF tended to be the highest (13.86\%). OFF also showed a high content, of $11.91 \%$, indicating that the maximum acteoside content can be obtained from flowers. The acteoside contents of the $100 \%$ and $80 \% \mathrm{EtOH}$ extract of OFVL were $10.81 \%$ and $11.14 \%$, respectively. In particular, if the leaves are superior to flowers in terms of acteoside content, further research on leaf extracts should be conducted. We found no existing reports on biologically active phytochemicals of $O$. fragrans leaves.

3.4. Antioxidant Properties of O. fragrans Extracts. The antioxidant activities of various extracts of $O$. fragrans were determined by measuring the DPPH-scavenging activity, reducing power, and total phenolic content. As shown in Figure 4, the extraction conditions produce higher DPPHscavenging activity in the $40 / 60 / 80 \% \mathrm{EtOH}$ for OFL and $60 \%$ $\mathrm{EtOH}$ for OFF than in the hot water.

The reducing power was tested for $100 \mu \mathrm{g} / \mathrm{mL}$ of each extract and was calculated as ascorbic acid equivalents ( $\mu \mathrm{g} / \mathrm{mL}$ eq. ascorbic acid). As shown in Figure 5 , the extraction condition produce higher reducing power in 
TABLE 3: Linearity and sensitivity of the present method.

\begin{tabular}{lccccc}
\hline Analyte & Retention time $(\mathrm{min})$ & $r^{2}$ & Linear range $(\mu \mathrm{g} / \mathrm{mL})$ & LOQ $(\mu \mathrm{g} / \mathrm{mL})$ & LOD $(\mu \mathrm{g} / \mathrm{mL})$ \\
\hline Acteoside & 12.28 & 0.999 & $6.25-100$ & 1.79 & 0.53 \\
\hline
\end{tabular}

TABLE 4: Intraday and interday precision and accuracy values for the present method $(n=3)$.

\begin{tabular}{|c|c|c|c|c|c|}
\hline \multirow{2}{*}{ Analyte } & \multirow{2}{*}{ Conc $(\mu \mathrm{g} / \mathrm{mL})$} & \multicolumn{2}{|c|}{ Intraday } & \multicolumn{2}{|c|}{ Inter-day } \\
\hline & & RSD (\%) & Accuracy (\%) & RSD (\%) & Accuracy (\%) \\
\hline \multirow{3}{*}{ Acteoside } & 12.5 & 0.30 & 98.33 & 1.12 & 98.10 \\
\hline & 25 & 0.32 & 102.59 & 0.27 & 102.75 \\
\hline & 50 & 0.20 & 103.42 & 0.52 & 103.27 \\
\hline
\end{tabular}

TABLE 5: Recovery of the present method $(n=6)$.

\begin{tabular}{lccc}
\hline Analyte & Added $(\mu \mathrm{g} / \mathrm{mL})$ & Recovery $(\%)(\mathrm{mean} \pm \mathrm{SD})$ & RSD $(\%)$ \\
\hline & 12.5 & $99.91 \pm 0.81$ & 0.76 \\
Acteoside & 25 & $100.74 \pm 1.30$ & 1.25 \\
& 50 & $103.56 \pm 0.41$ & 0.39 \\
\hline
\end{tabular}

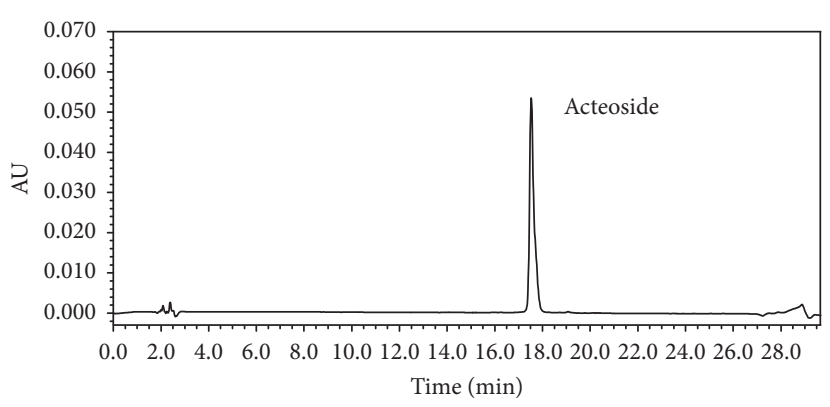

(a)

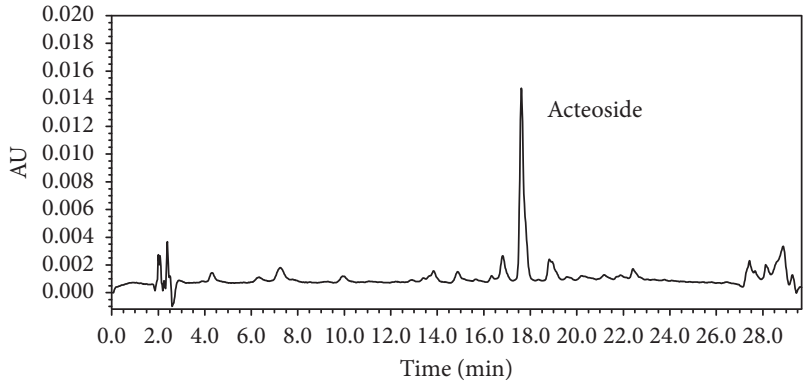

(b)

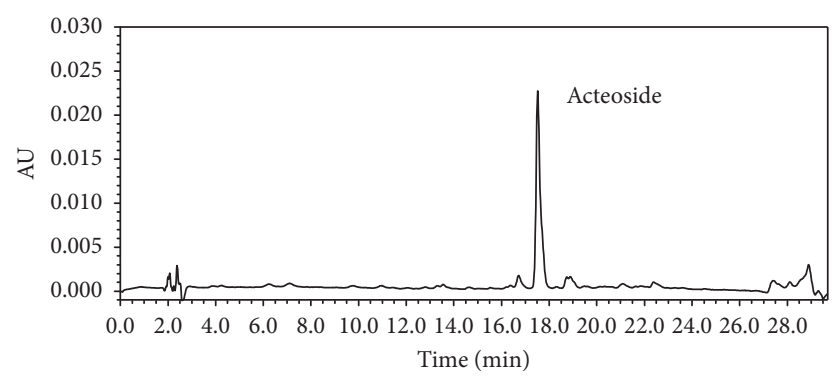

(c)

Figure 2: Typical high-performance liquid chromatography chromatograms of (a) acteoside standard (100 $\mu \mathrm{g} / \mathrm{mL}),(\mathrm{b})$ Osmanthus fragrans leaf $(0.2 \mathrm{mg} / \mathrm{mL})$, and (c) Osmanthus fragrans var. aurantiacus $(0.2 \mathrm{mg} / \mathrm{mL})$.

the $40 / 60 / 80 / 100 \% \mathrm{EtOH}$ for OFL, $60 / 80 / 100 \% \mathrm{EtOH}$ for OFF, $60 / 80 \% \mathrm{EtOH}$ for OFVL, and $60 / 80 \% \mathrm{EtOH}$ for OFVF than in the hot water. In particular, the 60 and $80 \%$ EtOH extracts of OFVL showed the best reducing power among all extracts $(176.83 \pm 4.53 \mu \mathrm{g} / \mathrm{mL}$ eq. ascorbic acid).

The total phenolic compounds, determined using the Folin-Ciocalteu method, were expressed as gallic acid equivalents, with reference to a standard curve $\left(r^{2}=0.999\right)$. The total phenolic contents of the extracts are shown in Figure 6. The extraction conditions produce higher total phenolic content in the $40 / 60 / 80 / 100 \% \mathrm{EtOH}$ for OFL, 60/80/100\% EtOH for OFF,
60/80/100\% EtOH for OFVL, and 60/80/100\% EtOH for OFVF. Particularly, the $80 \%$ EtOH extract of OFVL and the $100 \% \mathrm{EtOH}$ extract of OFL showed the highest total phenolic content. However, in terms of productivity, the extraction yield of the $100 \% \mathrm{EtOH}$ extract of OFL $(9.53 \%)$ was lower than that of the $80 \% \mathrm{EtOH}$ extract of OFVL (12.41\%). Thus, the $80 \%$ extract of OFVL showed the best phenolic content.

In summary, the flower and leaf extracts of $O$. fragrans and O. fragrans var. aurantiacus were prepared, and their extraction yields, marker compound (acteoside) content, and antioxidant activities were investigated. Overall, almost all extracts 


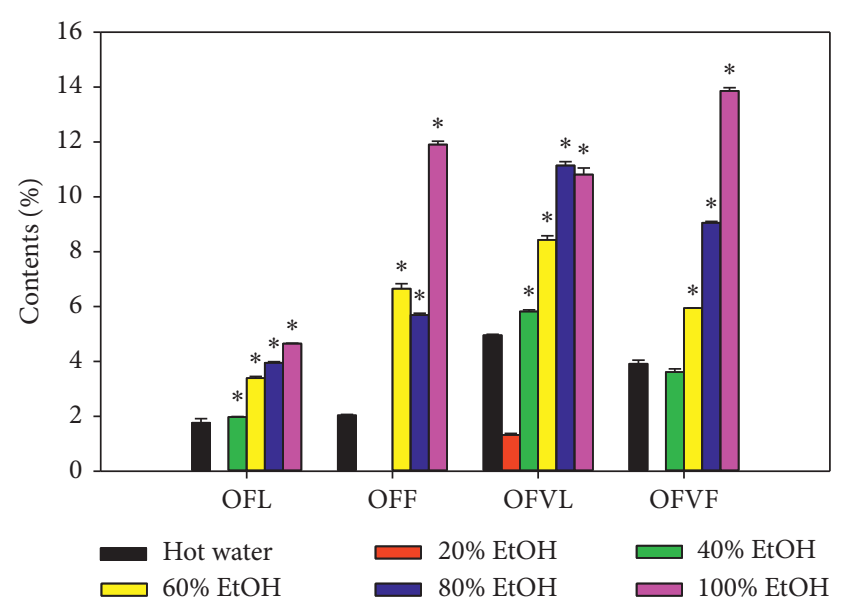

FIgURe 3: Acteoside contents in Osmanthus fragrans leaves (OFL), $O$. fragrans flowers (OFF), O. fragrans var. aurantiacus leaves (OFVL), and O. fragrans var. aurantiacus flowers (OFVF) $(n=3$; *significantly higher than the "hot water" extract).

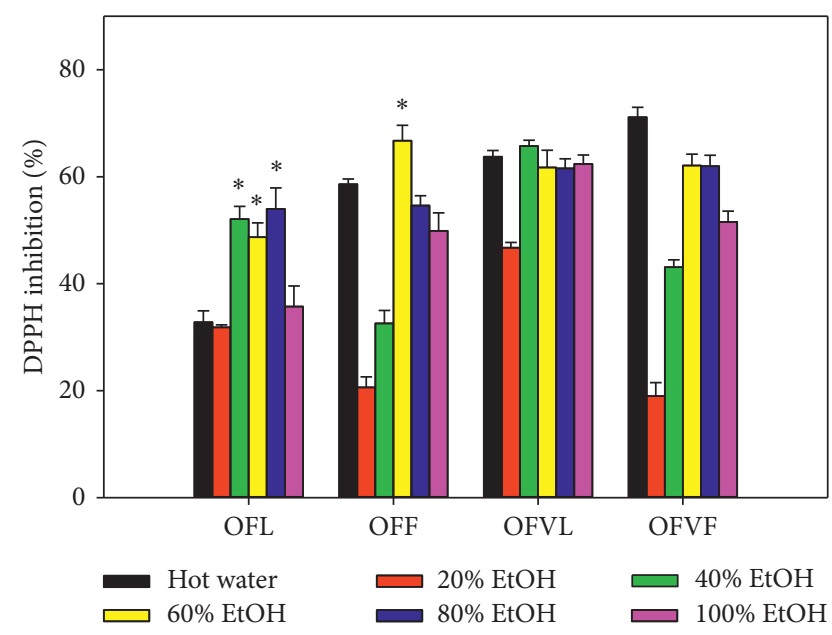

FIGURE 4: DPPH radical scavenging activity of various extracts of Osmanthus fragrans $\left(n=3\right.$; ${ }^{*}$ significantly higher than the "hot water" extract).

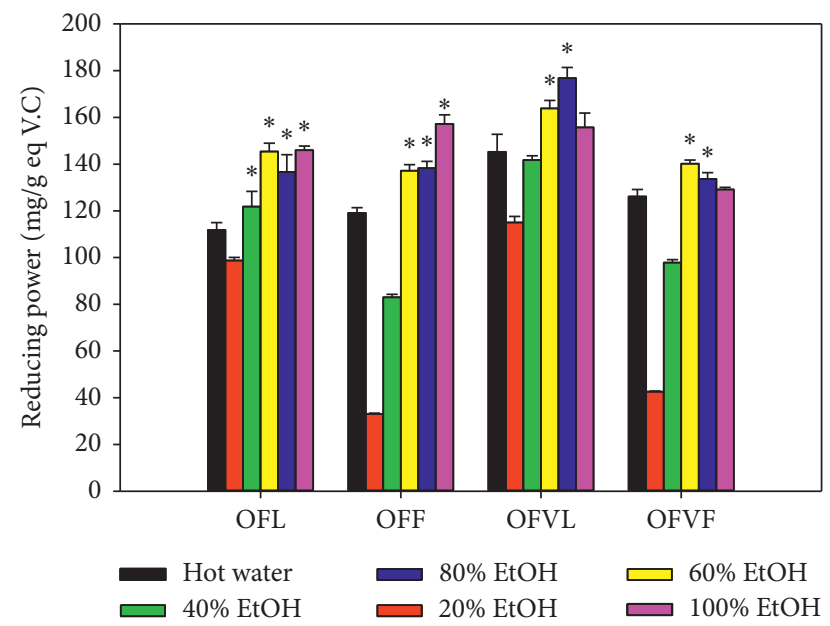

FIGURE 5: Reducing power of various extracts of Osmanthus fragrans ( $n=3$; *significantly higher than the "hot water" extract).

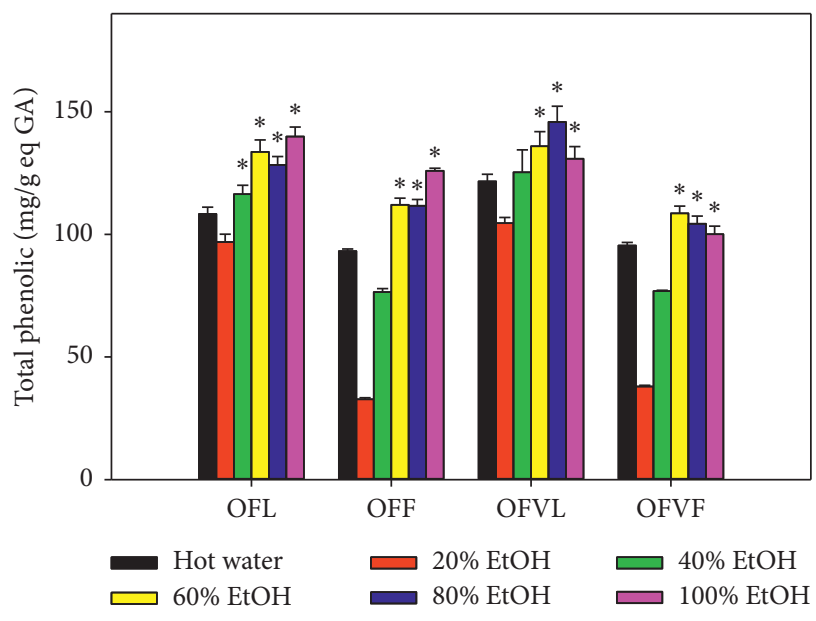

FIGURE 6: Total phenolics of various extracts of Osmanthus fragrans $\left(n=3 ;{ }^{*}\right.$ significantly higher than the "hot water" extract).

prepared using EtOH-based extraction solvents tended to produce better antioxidant activity than those prepared using hot water. These results suggest that the ethanolic extract of O. fragrans could serve as a potential antioxidant and antiinflammatory pharmaceutical source, and our validated method would be useful for the quality control of $O$. fragrans extracts.

\section{Data Availability}

The data used to support the findings of this study are available from the corresponding author upon request.

\section{Conflicts of Interest}

The authors declare that there are no conflicts of interest.

\section{Authors' Contributions}

Sung-Ho Lee and Seong-Wook Seo contributed equally to this work.

\section{Acknowledgments}

This study was carried out with the support of the "R\&D Program for Forest Science Technology (Project no. 2019141A00-2021-AB02)" provided by the Korea Forest Service (Korea Forestry Promotion Institute).

\section{References}

[1] B. Lu, M. Li, F. Zhou et al., “The Osmanthus fragrans flower phenylethanoid glycoside-rich extract: acute and subchronic toxicity studies," Journal of Ethnopharmacology, vol. 187, pp. 205-212, 2016.

[2] C. Y. Hung, F. L. Huang, L. S. Shi et al., "The ethanol extract of Osmanthus fragrans flowers reduces oxidative stress and allergic airway inflammation in an animal model," EvidenceBased Complementary and Alternative Medicine, vol. 2013, Article ID 304290, 10 pages, 2013.

[3] P. J. Tsai, M. L. Chang, C. M. Hsin, C.-C. Chuang, L.-T. Chuang, and W.-H. Wu, "Antilipotoxicity activity of 
Osmanthus fragrans and Chrysanthemum morifolium flower extracts in hepatocytes and renal glomerular mesangial cells," Mediators of Inflammation, vol. 2017, Article ID 4856095, 12 pages, 2017.

[4] F. Zhou, Y. Zhao, M. Li et al., "Degradation of phenylethanoid glycosides in Osmanthus fragrans Lour. flowers and its effect on anti-hypoxia activity," Scientific Reports, vol. 7, no. 1, Article ID 10068, 2017.

[5] H. Bin, C. Huangqin, and S. Longquan, "The ethanol extract of Osmanthus fragrans attenuates Porphyromonas gingivalis lipopolysaccharide-stimulated inflammatory effect through the nuclear factor erythroid 2-related factor-mediated antioxidant signalling pathway," Archives of Oral Biology, vol. 60, no. 7, pp. 1030-1038, 2015.

[6] H.-H. Lee, C.-T. Lin, and L.-L. Yang, "Neuroprotection and free radical scavenging effects of Osmanthus fragrans," Journal of Biomedical Science, vol. 14, no. 6, pp. 819-827, 2007.

[7] C.-Y. Hung, Y.-C. Tsai, and K.-Y. Li, "Phenolic antioxidants isolated from the flowers of Osmanthus fragrans," Molecules, vol. 17, no. 9, pp. 10724-10737, 2012.

[8] J. Liu, S. Nakamura, B. Xu et al., "Chemical structures of constituents from the flowers of Osmanthus fragrans var. aurantiacus," Journal of Natural Medicines, vol. 69, no. 1, pp. 135-141, 2015.

[9] H.-J. Choi, D.-H. Park, S.-H. Song, I.-S. Yoon, and S.-S. Cho, "Development and validation of a HPLC-UV method for extraction optimization and biological evaluation of hot-water and ethanolic extracts of dendropanax morbifera leaves," Molecules, vol. 23, no. 3, p. 650, 2018.

[10] Y.-H. Kim, M. Cho, D.-B. Kim et al., "The antioxidant activity and their major antioxidant compounds from Acanthopanax senticosus and A. koreanum," Molecules, vol. 20, no. 7, pp. 13281-13295, 2015. 